\title{
Teaching optics to a machine learning network
}

\author{
André-Pierre Blanchard-Dionne* and Olivier J. F. Martin $\mathbb{1}$ \\ Nanophotonics and Metrology Lab, École Polytechnique Fédérale de Lausanne, EPFL-STI-IMT-NAM, Station 11, 1015 Lausanne, Switzerland \\ ${ }^{*}$ Corresponding author: andre-pierre.blanchard-dionne@epfl.ch
}

Received 14 February 2020; revised 1 April 2020; accepted 20 April 2020; posted 20 April 2020 (Doc. ID 390600 ); published 15 May 2020

\begin{abstract}
In this Letter, we demonstrate how harmonic oscillator equations can be integrated in a neural network to improve the spectral response prediction for an optical system. We use the optical properties of a one-dimensional nanoslit array for a practical implementation of the study. This method allows to build more generalizable relations between the input parameters of the array and its optical properties, showing a 20-fold improvement for parameters outside the range used for the training. We also show how this model generates the output spectrum from phenomenological relationships between the input parameters and the output spectrum, indicating how it grasps the physical mechanisms of the optical response of the structure. (C) 2020 Optical Society of America
\end{abstract}

https://doi.org/10.1364/OL.390600

Machine-learning algorithms have been introduced recently in photonics as a tool to improve the design of nanostructures $[1,2]$. Such algorithms are based on artificial neural networks trained to perform a certain task, which is in the present case to determine the relationship between a structure's parameters and its optical properties. It has been proven a very powerful method, achieving high accuracy even for complex geometries, without the computational cost of running numerical simulations for every structure [3]. Furthermore, networks can be designed to do the opposite operation, for example, finding the right parameters to obtain specific optical properties, thus achieving the otherwise difficult task of reverse engineering [4-7]. Different networks have been demonstrated to work well for applications in optics, including the design of reflection coating [8], waveguides [9], optical storage units [10], power splitters [11], chemical sensors [12], as well as for the scattering of nanoparticles [13], circular dichroism in chiral metamaterials [14], or for color generation $[15,16]$. In this article, we draw inspiration from classical optics to introduce a method that improves the accuracy and the generalization potential of spectrum predictions using machine learning. Indeed, while regular networks can achieve great accuracy within the examples of the training set, this accuracy drops considerably for examples outside this range [17]. To circumvent this issue, we introduce neural networks that include harmonic oscillator equations, which are then used to generate a spectrum. This method is depicted in Fig. 1.

We demonstrate the advantage of using this approach for the transmission spectrum of a metallic grating in air with a glass substrate at normal incidence. The metallic grating is made of silver, and the thickness of the metal layer is fixed at $200 \mathrm{~nm}$. This structure with constant thickness can be describe with two parameters: namely, the period $d$ and the slit width a. This simple problem was chosen to clearly illustrate the advantage of the method in the case of optical spectrum experiments and to draw direct relations between the physics of the resonance and the oscillator parameters.

The idea we introduce in this model is to link the input parameters toward a summation of Lorentz oscillators that will fit the output spectrum of the structure. The Lorentzian equation can be written using four parameters [18]:

$$
l(\omega)=\frac{c e^{i \phi}}{\hbar\left(\omega-\omega_{0}\right)+i \gamma},
$$

where $c$ is a constant, $\phi$ is a phase factor, $\omega_{0}$ is the central resonance frequency, and $\gamma$ are the losses associated with the resonance. A Lorentzian oscillator was chosen since any optical resonance can be represented by this oscillator's equation or a combination of several such oscillators via the phase factor $\phi$ $[19,20]$. The total transmission for an optical system is given as

$$
t(\omega)=C_{t} e^{i \psi} \cdot \sum_{k} l_{k}(\omega),
$$

where $C_{t} e^{i \psi}$ corresponds to a background phase factor, which is also trained. The total network consists of five independent oscillator networks and the background. Each of these networks uses the nanoslit parameters a and $d$ as inputs, three fully connected hidden layers, and a layer with four nodes representing the four parameters of the oscillator, as shown in Fig. 2. A final output layer takes the sum of all oscillators multiplied by the background contribution to calculate both the real part and the imaginary part of the transmission coefficient $t$. This complex value spectrum is then compared with the grounded truth to calculate the loss function that is used to train the network.

For the training of the model, a dataset consisting of 10'167 complex spectra obtained with the coupled mode theory [21] was used for gratings with periods ranging in between $200 \mathrm{~nm}$ and $400 \mathrm{~nm}$ and slit widths ranging from $\mathrm{d} / 6$ to $\mathrm{d} / 3$. These parameters correspond to structures that can experimentally be realized with ease [22]. The thickness of the array was fixed at $200 \mathrm{~nm}$. The input training parameters were scaled in microns. The networks were developed using the Deep Learning library 


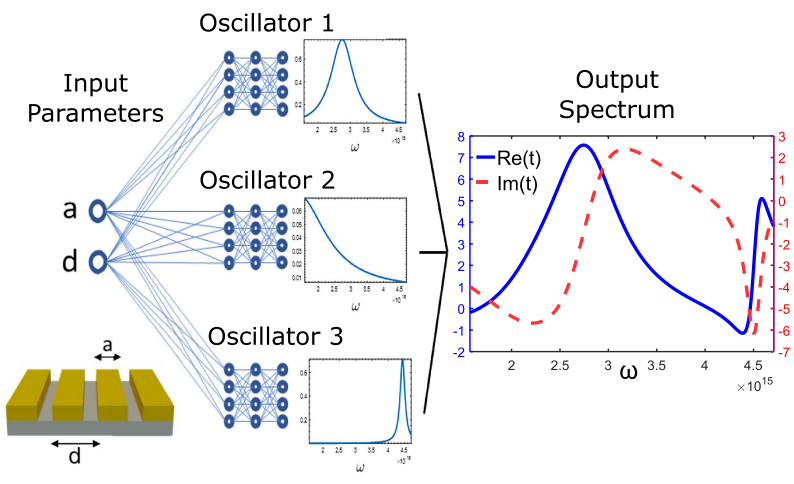

Fig. 1. Schematic of the neural network used. The grating is represented by the period $d$ and slit width $a$, which will serve as inputs for the different oscillators. Each oscillator consists in an independent network. The oscillator functions are summed to give an output spectrum, which is represented by the real and imaginary part of the transmission coefficient.

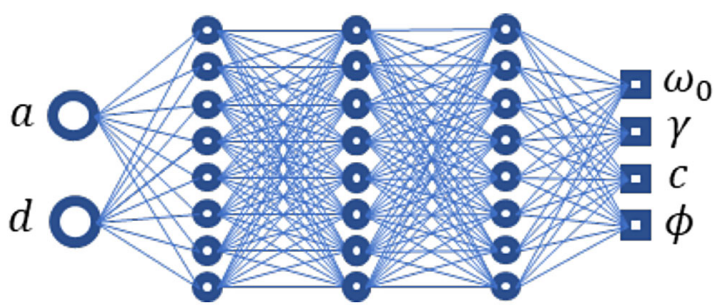

Fig. 2. Schematic of the oscillator network used. The network consists of fully connected layers between the input, three sets of hidden layers, and the output parameters of a Lorentzian distribution.

Keras under TensorFlow [23]. The hidden layers in each oscillator consist all of 250 nodes with a LeakyReLU activation function. The output layers of the oscillator used a "softplus" activation function for the two parameters $\omega$ and $\gamma$ to ensure positive physical values. An Adam optimizer was chosen with an adaptative learning rate that started at $l=1 \times 10^{-4}$ and was reduced by a factor of 0.75 every five epochs. The mean squared error of the concatenated real and imaginary parts of the transmission coefficient was used as the loss function. The neural network was trained on each set of parameters of the grating until the loss function of a validation set no longer improved, for about 60 epochs.

Once the network is trained, it can be used to predict the complex optical spectrum for any given set of parameters. A typical example is shown in Fig. 3 for a periodicity $d=266 \mathrm{~nm}$ and slit width $a=66 \mathrm{~nm}$. The predicted spectrum agrees very well with the target one, with a mean squared error reaching $\left\langle\sigma^{2}\right\rangle=3.6 \times 10^{-4}$. In Fig. 3(b), the output for each of the five oscillators is represented. Two sharp peaks (yellow and blue curves) accounts for resonances inside the array, which are due to extraordinary optical transmission [24], while the three other broad lines (green, purple, and red) contribute together with the background contribution $C_{t} e^{i \psi}$ to the transmission.

The use of the phase factor $\phi$ in Eq. (1) gives the possibility of interference between the different oscillators of the system and the background. For example, the extraordinary optical transmission exhibits a Fano-type resonance that originates from the interference between one spectrally narrow resonant
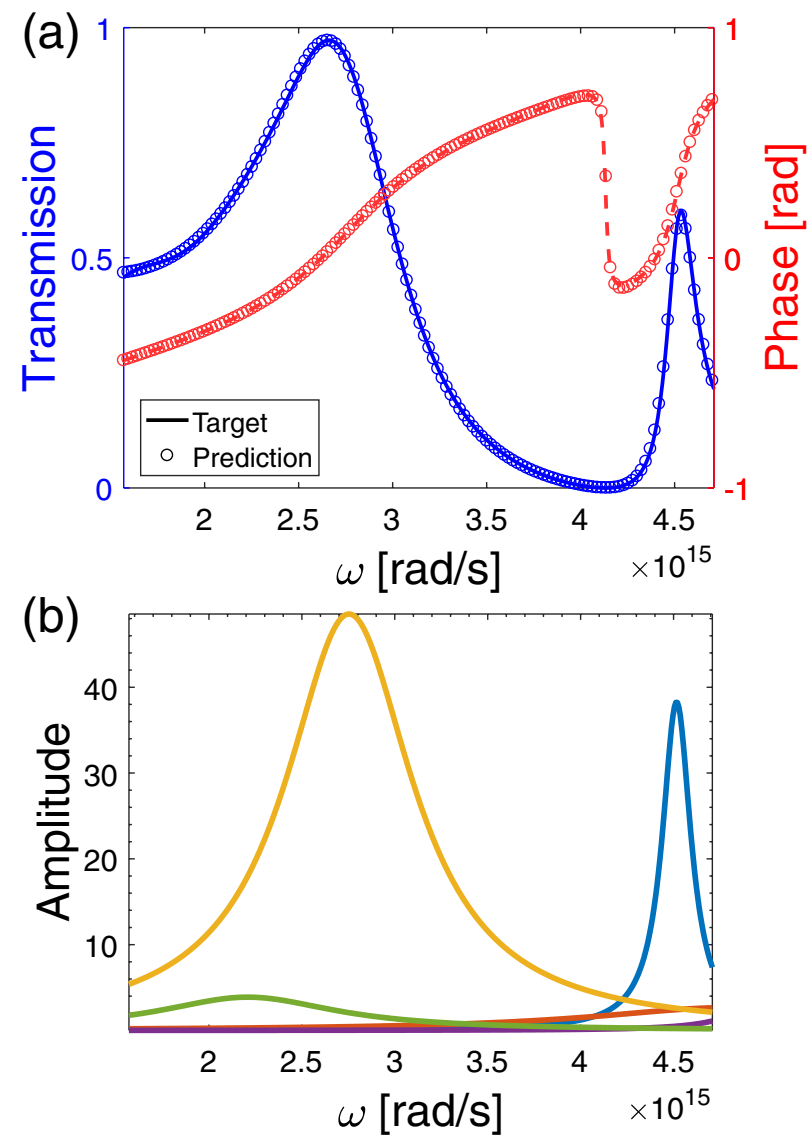

Fig. 3. (a) Predicted (circles) and target (full and dashed line) transmission and phase spectrum for a periodicity of $d=266 \mathrm{~nm}$ and a slit width of $a=66 \mathrm{~nm}$. (b) Output functions of each of the oscillators for the given parameters. Two curves (yellow and blue) show the resonant state of the grating array, while the other three curves (orange, green, and purple) contribute to the background function.

state and a broad background [25-27]. In Fig. 3(a), both resonance peaks show this asymmetrical line shape, which results from the summation of the individual resonant states (blue and yellow curves in Figure 3(b)) and the background (red, green, and purple curves in Figure 3(b)). The Lorentzian oscillators serve as elementary building blocks from which more complex resonances can emerge, providing more flexibility to the model to fit any type of optical resonance. One powerful aspect of this method is that the training draws a relationship between the input parameters of the structure and the optical resonances supported by it. This leads to a more stable and general link between the two since the function becomes phenomenological and follows the physical mechanisms of the resonance. Figure 4 shows the relationship between the output central frequency $\left(\omega_{0}\right)$ and loss $(\gamma)$ of the oscillator related to the first diffraction order resonance peak of the structure [orange curve in Fig. 3(a)] as a function of the periodicity for a fixed slit width.

The inverse of the central frequency, i.e., the central resonant wavelength, is plotted in Fig. 4(a) as a function of the period to highlight the linear relation that exists between the periodicity and the resonance wavelength. This relation remains monotonic and linear even for values outside the training set range, demonstrating the extrapolation capabilities of this method. The $\gamma$ factor shown in Fig. 4(b) for the same resonance peak 

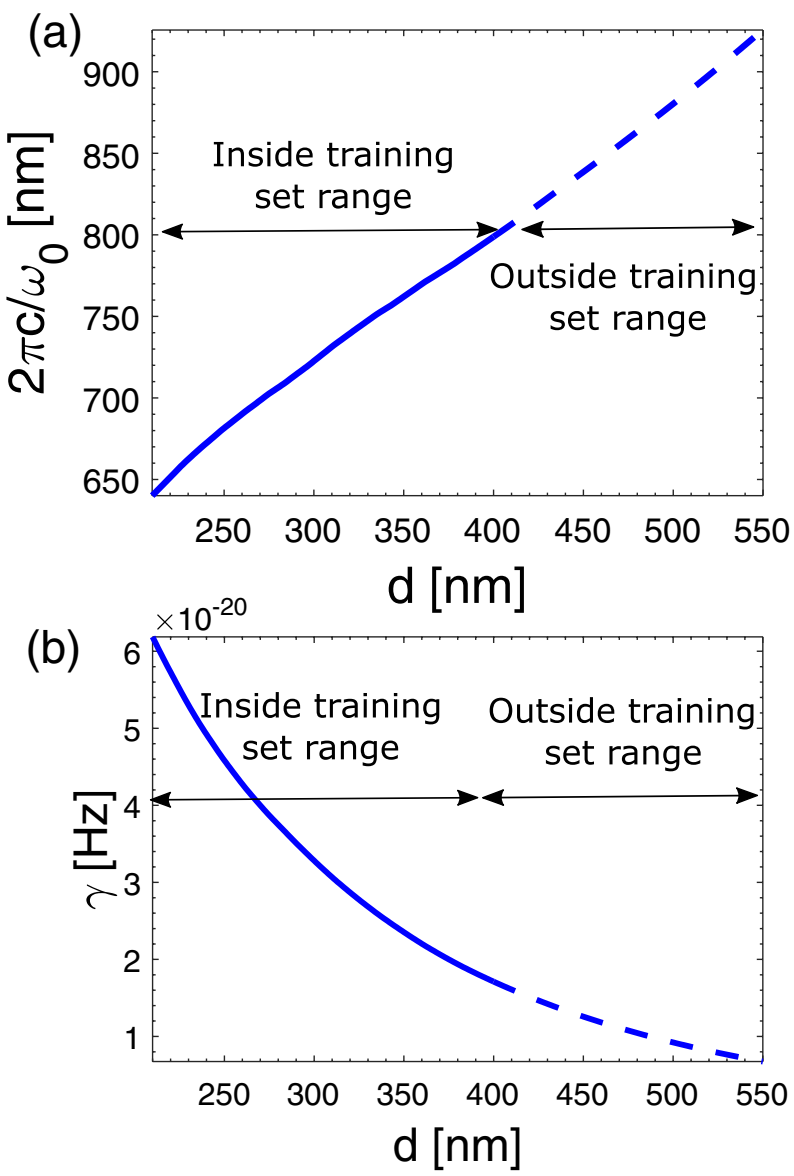

Fig. 4. Dependence of the parameters (a) $\omega_{0}$ and (b) $\gamma$ of the first diffraction order resonance of the slit array as a function of the periodicity for a fixed slit width $a=70 \mathrm{~nm}$.

also shows monotonic behavior in an inverse relation with the period, which is to be expected since the slits structure exhibits Fabry-Perot typed resonances for which the finesse is proportional to $1 / \mathrm{d}$ [21]. The conventional way to measure a model's accuracy in machine learning is to test it upon input parameters not used in the training. This is accomplished by choosing randomly a subgroup of parameters within the dataset along with their target spectrum, and by measuring the mean squared error of the prediction of this set that has not been "seen" by the model before. While this is sufficient to prove generality of the model within the values of the dataset, it does not indicate how well the model performs outside the range of the dataset values, or how well can the model think "outside the box." In the case of the model developed in this study, the periodicity was chosen between $200 \mathrm{~nm}$ and $400 \mathrm{~nm}$. We have also compared the optical responses for gratings with parameters outside this range and used them to assess whether the network would handle data outside this range, for values of periodicity ranging in between $400 \mathrm{~nm}$ and $550 \mathrm{~nm}$, and slit widths again ranging from $d / 6$ to $d / 3$. The prediction results for the oscillator model on this new set is presented in Fig. 5, alongside the results for a traditional neural network with direct spectrum output. This last model was simply a set of three hidden layers relating the input parameters to the output spectrum, with a similar number of parameters to the model with oscillators. The model with oscillators shows a much better representation of the desired spectrum in this case
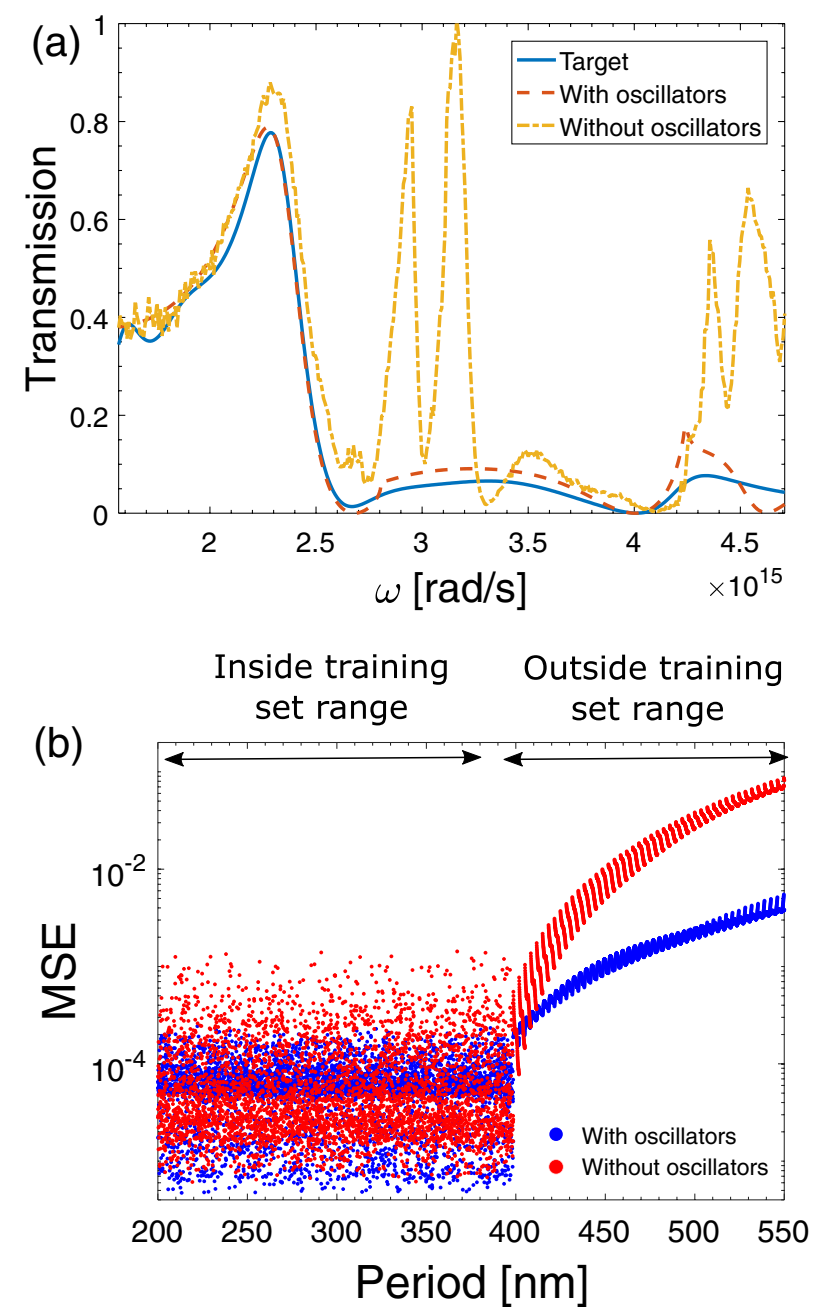

Fig. 5. (a) Target (blue curve) spectrum and predicted spectrum for a model with (red dashed curve) and without (yellow dashed-dotted curve) oscillators for parameters outside the range of the training set $(d=444 \mathrm{~nm}$ and $a=132 \mathrm{~nm})$. (b) Mean squared error (MSE) between the predicted spectrum and the target for values of periodicity ranging from values inside the training set $(200-400 \mathrm{~nm})$ and outside of it (400-500 nm).

since it is based upon the monotonic variation of the Lorentz parameters, which is more stable and robust, while the network without it shows spurious transmission variations.

Both networks behave well within the training set range with the average of the mean square error inside the training set range being $1.02 \times 10^{-4}$ and $6.01 \times 10^{-5}$, respectively, for the model without and with oscillators; this value goes to 0.031 and 0.0016 outside of this range, indicating an improvement of a factor of 20 when using the oscillator. While the model with direct spectrum points output becomes rather unreliable in that range, the model with oscillators shows a smoother transition with accurate predictions. The main limitation for this model arises when physical effects not represented in the training set start influencing the optical spectrum. In the case of the grating, higher diffraction orders start to emerge in the wavelength range chosen for this study, which is a limitation for any model built from machine learning. We can observe this difference in the high-frequency part of Fig. 5(a) where the small variations of the 
target (blue curve) are somewhat missed by the prediction (red dashed curve).

In this Letter, we have demonstrated that the inclusion of harmonic oscillators in the output of a neural network model improves its generalization ability by creating a more robust model. The study was conducted on the transmission spectrum of nanoslit arrays. The optical spectrum prediction shows an improvement of a factor of 20 compared to a traditional neural network with direct spectrum output for input data that lie outside the training set, demonstrating a more generalizable model. This improvement comes from the parametrization of the output, which includes phenomenological relations with the parameters of the slit array.

Funding. Quebec Fonds de Recherche Nature et Technologies (B3 259466); European Research Council (ERC-2015-AdG-695206 Nanofactory).

Disclosures. The authors declare no conflicts of interest.

\section{REFERENCES}

1. K. Yao, R. Unni, and Y. Zheng, Nanophotonics 8, 339 (2019).

2. J. Zhou, B. Huang, Z. Yan, and J.-C. G. Bünzli, Light. Sci. Appl. 8, 1 (2019).

3. I. Sajedian, J. Kim, and J. Rho, "Predicting resonant properties of plasmonic structures by deep learning," arXiv:1805.00312 (2018).

4. W. Ma, F. Cheng, Y. Xu, Q. Wen, and Y. Liu, "Probabilistic representation and inverse design of metamaterials based on a deep generative model with semi-supervised learning strategy," arXiv:1901.10819 (2019).

5. D. Liu, Y. Tan, E. Khoram, and Z. Yu, ACS Photon. 5, 1365 (2018).

6. S. So and J. Rho, Nanophotonics 8, 1255 (2019).

7. P. M. Tagade, S. P. Adiga, S. Pandian, M. S. Park, K. S. Hariharan, and S. M. Kolake, npj Comput. Mater. 5, 1 (2019).
8. Z. Liu, D. Zhu, S. P. Rodrigues, K.-T. Lee, and W. Cai, Nano Lett. 18 6570 (2018).

9. T. Zhang, J. Wang, Q. Liu, J. Zhou, J. Dai, X. Han, Y. Zhou, and K. Xu, Photon. Res. 7, 368 (2019).

10. P. R. Wiecha, A. Lecestre, N. Mallet, and G. Larrieu, Nat. Nanotechnol. 14, 237 (2019).

11. M. H. Tahersima, K. Kojima, T. Koike-Akino, D. Jha, B. Wang, C. Lin, and K. Parsons, "Deep neural network inverse design of integrated nanophotonic devices," arXiv:1809.03555 (2018).

12. I. Malkiel, M. Mrejen, A. Nagler, U. Arieli, L. Wolf, and H. Suchowski, Light Sci. Appl. 7, 60 (2018).

13. J. Peurifoy, Y. Shen, L. Jing, Y. Yang, F. Cano-Renteria, B. G. DeLacy, J. D. Joannopoulos, M. Tegmark, and M. Soljačić, Sci. Adv. 4, eaar4206 (2018).

14. W. Ma, F. Cheng, and Y. Liu, ACS Nano 12, 6326 (2018).

15. J. Baxter, A. C. Lesina, J.-M. Guay, A. Weck, P. Berini, and L. Ramunno, Sci. Rep. 9, 8074 (2019).

16. I. Sajedian, T. Badloe, and J. Rho, "Finding the best design parameters for optical nanostructures using reinforcement learning," arXiv:1810.10964 (2018).

17. R. O. Malashin and A. B. Kadykov, J. Opt. Technol. 82, 509 (2015).

18. A. F. J. Levi, Essential Classical Mechanics for Device Physics (Morgan \& Claypool, 2016), pp. 2053-2571, pp. 5-1 to 5-21.

19. A. Christ, Y. Ekinci, H. Solak, N. Gippius, S. Tikhodeev, and O. Martin, Phys. Rev. B 76, 201405 (2007).

20. M. F. Limonov, M. V. Rybin, A. N. Poddubny, and Y. S. Kivshar, Nat. Photonics 11, 543 (2017).

21. A.-P. Blanchard-Dionne and M. Meunier, Adv. Opt. Photon. 9, 891 (2017).

22. G. Quaranta, G. Basset, O. J. Martin, and B. Gallinet, ACS Photon. 4, 1060 (2017).

23. F. Chollet, "Keras" 2015, https://keras.io.

24. T. W. Ebbesen, H. J. Lezec, H. Ghaemi, T. Thio, and P. A. Wolff, Nature 391, 667 (1998).

25. C. Genet, M. P. van Exter, and J. Woerdman, Opt. Commun. 225, 331 (2003).

26. B. Gallinet and O. J. Martin, Phys. Rev. B 83, 235427 (2011).

27. C. Yan, K.-Y. Yang, and O. J. Martin, Light Sci. Appl. 6, e17017 (2017). 